Check for updates

Cite this: RSC Adv., 2017, 7, 22548

\title{
Electrospun nanofibrous membrane of porous fluorine-containing triptycene-based polyimides for oil/water separation $\uparrow$
}

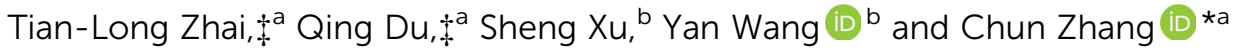 \\ A novel nanofibrous membrane for oil/water separation is reported. Electrospinning was employed to \\ fabricate the nanofibrous membrane from a porous polymer of fluorine-containing triptycene-based \\ polyimide (FTPI), which was synthesized and characterized by NMR, FT-IR and TGA. With porous \\ properties and highly hydrophobic features, the membrane of FTPI showed a large water contact angle \\ (WCA) $\left(121^{\circ}\right)$, high flux and great separation efficiency for oil/water mixtures. We believe that the fluorine- \\ contained nanofibrous membranes may be considered as promising candidates for oil/water separation.
}

Received 8th February 2017

Accepted 19th April 2017

DOI: $10.1039 / c 7 r a 01614 j$

rsc.li/rsc-advances can more overcome the flux limitations because of their advantages of higher porosity, lower base weight, larger effective surface area, and continuously interconnected pores. Recently, some electrospun membranes have been developed successfully for oil/water separation from polymeric/inorganic silica hybrid systems. ${ }^{21,22}$ Polymers of intrinsic microporosity (PIMs), ${ }^{23-25}$ the only one solution processible porous organic polymer, have not been applied widely in the field of electrospun nanofibrous membrane for oil/water separation yet, although they have a lot of advantages, such as high surface area, low mass density, easy functionality and high stability.

Triptycene and its derivatives have attractive applications in supramolecular chemistry ${ }^{26-29}$ and microporous polymers ${ }^{30-33}$ because of their 3D rigid structures. More and more triptycene based PIMs ${ }^{34-36}$ are being reported in gases separation, but not in oil/water separation. It is generally accepted that fluorochemicals provide a new approach to fabricate the superhydrophobic materials. ${ }^{21,37,38}$ Fluorine-containing triptycenebased polyimide (FTPI), one kind of PIMs, have not been reported in fields of electrospun nanofibrous membrane for oil/ water separation, although aromatic polyimides ${ }^{39-41}$ have been recognized as a great potential materials in fields of porous materials with the features of low density, thermal stability, high glass transition temperature, chemical resistance and good mechanical properties. Herein, we report the fabrication of high-hydrophobic electrospun nanofibrous membrane based on FTPI (Scheme 1) for oil/water separation with high flux and great separation efficiency.

\section{Experimental}

\section{Materials and methods}

2,6-Diaminotriptycene was synthesized according to ref. 42, 4,4'-(hexafluoroisopropylidene) diphthalic anhydride (6FDA) was purchased from TCI (Shanghai) Development Co. Ltd. 

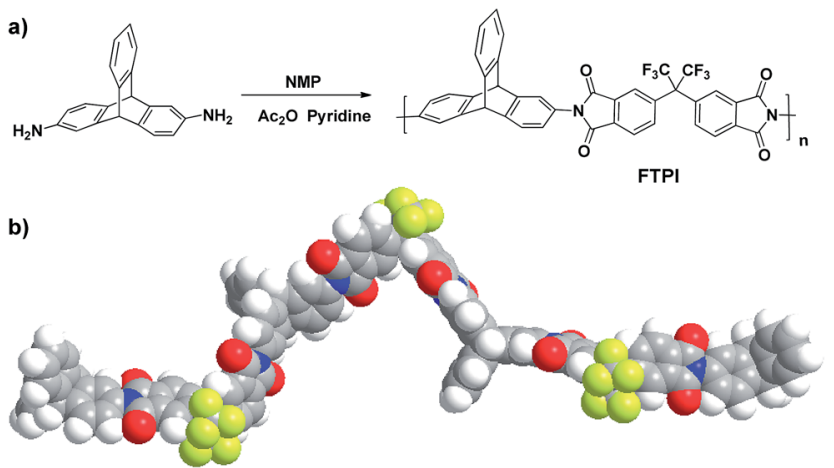

Scheme 1 (a) The synthesis and structure of FTPI. (b) A molecular model of FTPI showing its contorted shape, which combined with its rigidity generates intrinsic microporosity due to an inability to pack efficiently in the solid state.

Other reagents from Sinopharm Chemical Reagents are of analytical grade and used without further purification.

${ }^{1} \mathrm{H}$ nuclear magnetic resonance (NMR) spectra were recorded on Bruker model AV600 NMR spectrometers, where chemical shifts were determined with TMS. Fourier transform infrared (FT-IR) spectra were recorded on a Bruker model VERTEX 70 infrared spectrometer. Thermogravimetric analysis (TGA) measurements were performed on a PerkinElmer model Pyris1 TGA under $\mathrm{N}_{2}$, by heating to $800{ }^{\circ} \mathrm{C}$ at a rate of $10{ }^{\circ} \mathrm{C} \mathrm{min}^{-1}$. Field-emission scanning electron microscopy (FE-SEM) measurements were performed on Tescan VEGA 3 SBH field-emission scanning electron microscope. Average diameter of fibers were measured and calculated using Image J (National Institutes of Health (NIH), USA) based on SEM images. The breaking strength of the nanofibrous membrane was tested by electronic universal testing machine (CMT 4041, Shenzhen SANS Testing Machine Co., Ltd). Water contact angle (WCA) studies were conducted on Kruss ZSA25 Contact Angle Goniomete. Electrospinning nanofibrous membrane were carried out at a SS-2535H equipment, which purchased from Beijing Ucalery Technology and Development Co. Ltd.

\section{Synthesis of FTPI}

To a mixed solution of 2,6-diaminotriptycene (4.0 mmol), 6FDA $(4.0 \mathrm{mmol})$ in $N$-methylpyrrolidone (NMP) $(20 \mathrm{~mL})$ was added. The reaction mixture was stirred at the room temperature for one day to form a viscous liquid. Pyridine $(2.0 \mathrm{~mL})$ and acetic anhydride $(4.0 \mathrm{~mL})$ were added to the above reaction. The resulting mixture was stirred at room temperature for an additional $1 \mathrm{~h}$ and then it was heated to $110{ }^{\circ} \mathrm{C}$ for one day. After cooling to room temperature, the reaction mixture was poured into methanol $(200 \mathrm{~mL})$. The precipitate was collected by filtration and washed thoroughly with methanol. Further purification involved the precipitation of a THF solution in methanol, Soxhlet extraction with methanol for 2 days, and a vacuum drying at $60{ }^{\circ} \mathrm{C}$, leading to solids as the product FTPI with yield of $88.6 \%$.

\section{Electrospinning of polyimide nanofibrous membrane}

Typically, FTPI was dissolved in $N, N$-dimethylformamide to form a polymer solution at a concentration of $60 \%(\mathrm{w} / \mathrm{v})$. The polymer solution was loaded into a syringe with a 22 -Gauge spinneret (inner diameter: $0.41 \mathrm{~mm}$ ). A grounded aluminum foil was used as a collector, which was $16 \mathrm{~cm}$ below the spinneret. The voltage between the spinneret and the collector was maintained at $14 \mathrm{kV}$. The feeding rate was controlled by a syringe pump at a constant rate of $0.5 \mathrm{~mL} \mathrm{~h}^{-1}$. This procedure carried out for $1 \mathrm{~h}$. The electrospun membranes were then dried under vacuum at $40{ }^{\circ} \mathrm{C}$ for $12 \mathrm{~h}$.

\section{Oil/water separation}

To assess the oil/water separation performance of the nanofibrous membrane, we employed the flux $(F)$ and separation efficiency as the key parameters. The experiments of flux and separation efficiency were carried out by four kinds oils, and the separation efficiency $(\eta)$ was calculated following the work of Ma et al. ${ }^{43}$

$$
\eta=\frac{V_{1}}{V_{0}} \times 100
$$

where $V_{1}$ and $V_{0}$ are the volume of water recovered after separation and before separation process, respectively.

\section{Results and discussion}

FTPI was synthesized according to Swager's strategy. ${ }^{42}$ Typically, diaminotriptycene and 6FDA were dissolved in NMP and gave the poly(amic acid), the chemical dehydrating agents (pyridine and acetic anhydride) were added subsequently to fabricated FTPI in yield of $88.6 \%$. The structure of FTPI was characterized by ${ }^{1} \mathrm{H}$ NMR, FT-IR, TGA and GPC. The ${ }^{1} \mathrm{H}$ NMR spectrum (Fig. S1, ESI $\dagger$ ) showed a singlet signal in $5.53 \mathrm{ppm}$, corresponding to the bridgehead protons of triptycene moieties. Moreover, there are not any proton signals for the carboxylic acid in the spectrum, suggesting complete imidization. The FTIR spectrum of FTPI exhibited typical vibration features for a polyimide, as shown in Fig. 1a and b, the symmetric and asymmetric stretching bands of the imide carbonyl were observed at 1726 and $1786 \mathrm{~cm}^{-1}$ for FTPI, respectively. Moreover, the absorption of the $\mathrm{C}-\mathrm{N}-\mathrm{C}$ group could be detected at $1373 \mathrm{~cm}^{-1}$. These results provided evidence for the formation of the five-membered imide rings.

Just like other polyimides, the thermogravimetric analysis (TGA) (Fig. S2, ESI $\dagger$ ) suggested that FTPI was stable up to $500^{\circ} \mathrm{C}$. The gel permeation chromatography (GPC) data displayed that the molecular weight $\left(M_{\mathrm{n}}\right)$ were $41 \mathrm{kDa}$ with polydispersity index (PDI) of 1.17 for FTPI. Solution viscosity measurements by Ubbelohde type viscometer in NMP $\left(0.38 \mathrm{dL}^{-1}\right)$ reveal extremely low inherent viscosities, which is similar to other triptycene-containing polyimides. ${ }^{42,44}$ The porous properties of FTPI were studied by nitrogen sorption analysis at $77 \mathrm{~K}$. The Brunauer-Emmett-Teller (BET) (Fig. S3, ESI $\dagger$ ) surface area is $202 \mathrm{~m}^{2} \mathrm{~g}^{-1}$ (the Langmuir surface area is $280 \mathrm{~m}^{2} \mathrm{~g}^{-1}$ ) and the total volume is $0.204 \mathrm{~cm}^{3} \mathrm{~g}^{-1}$. The nitrogen sorption isotherm 

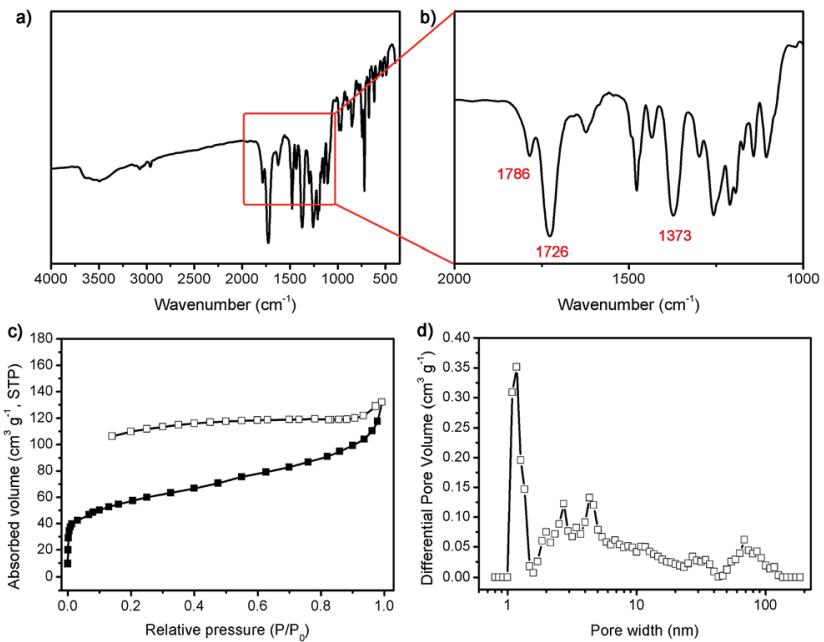

Fig. 1 FT-IR spectrum (a) and (b), nitrogen sorption isotherm at $77 \mathrm{~K}(\mathrm{c})$ and pore size distribution (d) of FTPI.

for FTPI is shown in Fig. 1c, a steep nitrogen gas uptake at low relative pressure $\left(P / P_{0}<0.001\right)$ indicates a large amount of microporous structure. The hysteresis was associated with the irreversible uptake of gas molecules in the pores (or through pore entrances), which was probably attributed to network swelling. The pore size distribution calculated using nonlocal density functional theory (NLDFT) method (Fig. 1d) also confirmed the presence of primary micropore and a spot of meso- and macropore.

With good porous properties, FTPI was dissolved in DMF and used to prepared nanofibrous membranes by electrospinning. When the concentration of electrospun solution was lower than $60 \%$, beaded fibers were formed (Fig. S4, ESI $\dagger$ ). If it was higher than $60 \%$, the spinneret was tended to be blocked due to the high viscosity. So the concentration of electrospun solution was chosen to be $60 \%$. At this condition, uniform fibers with a diameter of $707( \pm 131) \mathrm{nm}$ were achieved as shown in Fig. 2a and b, and the distribution diagram of fiber diameters was shown in ESI (Fig. S5†). SEM images also showed that the pores of the membrane consisted by the nanofibers were with a size of several micrometers. The surface of the membrane was smooth (Fig. S6, ESI $\dagger$ ) and its thickness was about $140 \mu \mathrm{m}$ calculated from the SEM image of the cross-section (Fig. S7, ESI $\dagger$ ). Stress-strain behaviour of FTPI nanofibrous membrane was tested and the tensile strength of $0.56 \mathrm{MPa}$ may be owed to the low molecular weight of FTPI (Fig. S8, ESI $\dagger$ ).
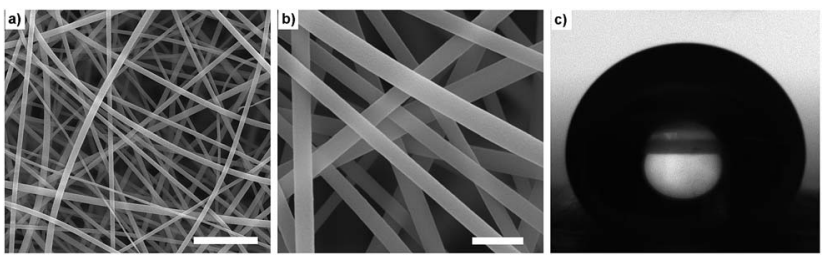

Fig. 2 (a) and (b) FE-SEM images of the nanofibrous membrane (scale bar: $10 \mu \mathrm{m}$ and $2 \mu \mathrm{m}$ for (a) and (b), respectively). (c) Photographs of a water droplet on FTPI membrane.
The surface wettability of the FTPI nanofibrous membrane has been characterized by the water contact angle (WCA) measurement. Fig. 2c displays the high-hydrophobic for FTPI membrane with WCA of $121^{\circ}$. Compared with the WCA $\left(<80^{\circ}\right)$ for other fluorine-free polyimides membranes, ${ }^{\mathbf{4 3 , 4 5}}$ the higher WCA of FTPI membrane may be owe to the abundant fluorine element.

With high-hydrophobic property, the FTPI membrane was carried out for oil/water separation. As shown in Fig. 3a and b, the mixture of oil/water was poured onto the nanofibrous membrane, which was fixed between two glass tubes and placed a tilt angle of $20^{\circ}$. It was observed clearly that the $n$-hexane will float above water, because of lower density. Therefore, tilting slightly made sure that $n$-hexane could contact with the nanofibrous membrane. We have controlled the volume of water to keep organic phase contacting with nanofibrous membrane. The mixture of $n$-hexane ( $20 \mathrm{~mL}$, dyed by Sudan III) and water (10 $\mathrm{mL}$, dyed by methylene blue) was poured into the upper glass tube. The obvious results were that oil phase permeates through membrane by the driving force of gravity while water still stayed on the upper glass tube (see more details in the ESI video $\dagger$ ). As presented in Fig. 3c, the separation efficiency of membrane for different oils is about $99 \%$. The reuse ability of FTPI nanofibrous membrane was further examined in the successive separation of $n$-hexane/water mixture. As shown in Fig. 3d, the high separation efficiency can be kept after 10 successive runs. Furthermore, we added two different electrospinning duration, $0.5 \mathrm{~h}$ and $4.0 \mathrm{~h}$, with the same electrospinning condition. According to the experiment results, membranes with different thickness all displayed great separation efficiency (ESI, Fig. S9†). These results suggest the FTPI nanofibrous membrane has excellent separation efficiency for the oil/water mixture.

To test the flux, four kinds of organic solvents $(30 \mathrm{~mL})$ were permeated through membranes with different electrospinning
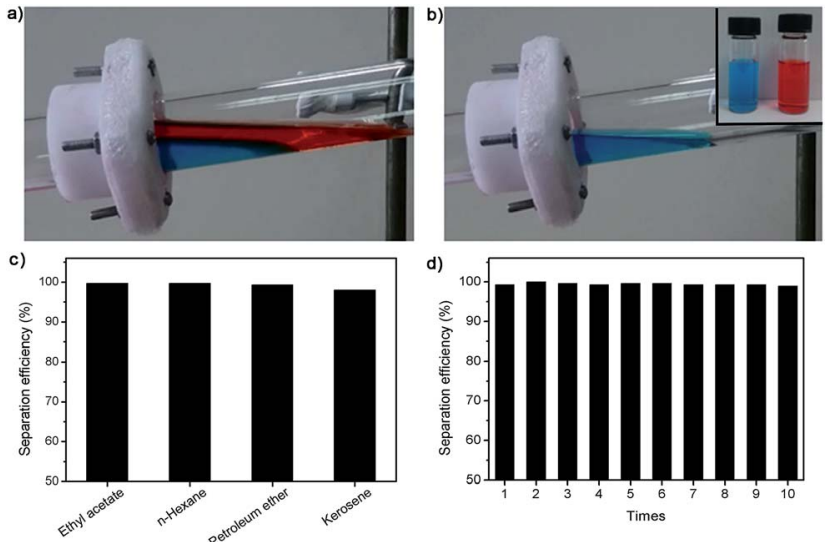

Fig. 3 ( $a$ and b) Oil/water separation process. The mixture of $n$ hexane and water was poured into the upper glass tube, oil phase permeated the FTPI membrane by the force of gravity. Collecting the water and $n$-hexane after separation process (inserting picture of b). (c) The separation efficiency of nanofibrous membrane for different oils. (d) The separation efficiency of hexane and water kept high level after 10 successive runs. 
durations (ESI, Fig. S10 $\dagger$ ), and the flux $(F)$ was calculated according to the equation:

$$
F=\frac{V}{A \Delta t}
$$

where $V(\mathrm{~L})$ is the volume of organic solvents which pass through the membrane, $A\left(\mathrm{~m}^{2}\right)$ and $\Delta t(\mathrm{~h})$ are the area of the membrane and the filtration time, respectively. The flux values of membrane decreased for petroleum ether, $n$-hexane, ethyl acetate and kerosene, respectively. Those results are consistent with the inversely proportional relationship between flux and kinetic viscosity. It is worth noting that the flux values are significantly higher than the (typical) flux through commercial filtration membranes $\left(20-200 \mathrm{~L} \mathrm{~m}^{-2} \mathrm{~h}^{-1}\right) .{ }^{46}$

\section{Conclusion}

In summary, we have synthesized a triptycene based fluorinated polyimide and confirmed its structure by NMR, FT-IR and TGA. Moreover, the nanofibrous membranes were fabricated from FTPI by electrospinning. With the high-hydrophobic feature, the membrane of FTPI showed large WCAs, high flux and great separation efficiency. We believe that the fluorine-contained nanofibrous membrane may be considered as a promising candidate for oil/water separation.

\section{Acknowledgements}

This work is supported by the National Natural Science Foundation of China (21672078), the Natural Science Foundation of Hubei Province (2016CFB372), the Applied Basic Program of Wuhan City (2016010101010017) and the Fundamental Research Funds for the Central Universities (HUST 2015TS086). We thank the Analytical and Testing Center of Huazhong University of Science and Technology for related analysis.

\section{References}

1 B. Wang, W. Liang, Z. Guo and W. Liu, Chem. Soc. Rev., 2015, 44, 336-361.

2 J.-T. Wang, Y. Zheng and A.-Q. Wang, Chem. Eng. J., 2012, 213, 1-7.

3 Y. Zheng, J.-T. Wang, Y.-F. Zhu and A.-Q. Wang, J. Environ. Sci., 2015, 27, 21-32.

4 Q. F. Wei, R. R. Mather, A. F. Fotheringham and R. D. Yang, Mar. Pollut. Bull., 2003, 46, 780-783.

5 H. T. T. Duong and R. P. Burford, J. Appl. Polym. Sci., 2006, 99, 360-367.

6 M. A. Lillo-Ródenas, D. Cazorla-Amorós and A. LinaresSolano, Carbon, 2005, 43, 1758-1767.

7 Q. Zhu, Q. Pan and F. Liu, J. Phys. Chem. C, 2011, 115, 1746417470.

8 L. Shi, K. Chen, R. Du, A. Bachmatiuk, M. H. Rummeli, K. Xie, Y. Huang, Y. Zhang and Z. Liu, J. Am. Chem. Soc., 2016, 138, 6360-6363.

9 J. Wang, Z. Shi, J. Fan, Y. Ge, J. Yin and G. Hu, J. Mater. Chem., 2012, 22, 22459-22466.
10 Z. Niu, J. Chen, H. H. Hng, J. Ma and X. Chen, Adv. Mater., 2012, 24, 4144-4150.

11 H.-P. Cong, X.-C. Ren, P. Wang and S.-H. Yu, ACS Nano, 2012, 6, 2693-2703.

12 Y. Cao, X. Zhang, L. Tao, K. Li, Z. Xue, L. Feng and Y. Wei, ACS Appl. Mater. Interfaces, 2013, 5, 4438-4442.

13 M. Padaki, R. Surya Murali, M. S. Abdullah, N. Misdan, A. Moslehyani, M. A. Kassim, N. Hilal and A. F. Ismail, Desalination, 2015, 357, 197-207.

14 R. Wanga, Y. Liua, B. Li, B. S. Hsiaoa and B. Chua, J. Membr. Sci., 2012, 392, 167-174.

15 Y. Cao, N. Liu, W. Zhang, L. Feng and Y. Wei, ACS Appl. Mater. Interfaces, 2016, 8, 3333-3339.

16 T. Zhao, D. Zhang, C. Yu and L. Jiang, ACS Appl. Mater. Interfaces, 2016, 8, 24186-24191.

17 A. Greiner and J. H. Wendorff, Angew. Chem., Int. Ed., 2007, 46, 5670-5703.

18 K. Acatay, E. Simsek, C. Ow-Yang and Y. Z. Menceloglu, Angew. Chem., Int. Ed., 2004, 43, 5210-5213.

19 D. Li and Y. Xia, Adv. Mater., 2004, 16, 1151-1170.

20 W. Ma, Q. Zhang, D. Hua, R. Xiong, J. Zhao, W. Rao, S. Huang, X. Zhan, F. Chen and C. Huang, RSC Adv., 2016, 6, 12868-12884.

21 Y. Shang, Y. Si, A. Raza, L. Yang, X. Mao, B. Ding and J. Yu, Nanoscale, 2012, 4, 7847-7854.

22 M. Obaid, G. M. K. Tolba, M. Motlak, O. A. Fadali, K. A. Khalil, A. A. Almajid, B. Kim and N. A. M. Barakat, Chem. Eng. J., 2015, 279, 631-638.

23 N. B. McKeown and P. M. Budd, Chem. Soc. Rev., 2006, 35, 675-683.

24 N. B. McKeown and P. M. Budd, Macromolecules, 2010, 43, 5163-5176.

25 M. Carta, R. Malpass-Evans, M. Croad, Y. Rogan, J. C. Jansen, P. Bernardo, F. Bazzarelli and N. B. McKeown, Science, 2013, 339, 303-307.

26 Y. Han, Z. Meng, Y.-X. Ma and C.-F. Chen, Acc. Chem. Res., 2014, 47, 2026-2040.

27 C.-F. Chen, Chem. Commun., 2011, 47, 1674-1688.

28 Z. Meng, Y. Han, L.-N. Wang, J.-F. Xiang, S.-G. He and C.-F. Chen, J. Am. Chem. Soc., 2015, 137, 9739-9745.

29 G.-W. Zhang, P.-F. Li, Z. Meng, H.-X. Wang, Y. Han and C.-F. Chen, Angew. Chem., Int. Ed., 2016, 55, 5304-5308.

30 T.-L. Zhai, L. Tan, Y. Luo, J.-M. Liu, B. Tan, X.-L. Yang, H.-B. Xu and C. Zhang, Chem.-Asian J., 2016, 11, 294-298.

31 H. Ma, J.-J. Chen, L. Tan, J.-H. Bu, Y. Zhu, B. Tan and C. Zhang, ACS Macro Lett., 2016, 5, 1039-1043.

32 C. Zhang, P.-C. Zhu, L. Tan, J.-M. Liu, B. Tan, X.-L. Yang and H.-B. Xu, Macromolecules, 2015, 48, 8509-8514.

33 C. Zhang, J.-J. Wang, Y. Liu, H. Ma, X.-L. Yang and H.-B. Xu, Chem.-Eur. J., 2013, 19, 5004-5008.

34 M. Carta, M. Croad, R. Malpass-Evans, J. C. Jansen, P. Bernardo, G. Clarizia, K. Friess, M. Lanč and N. B. McKeown, Adv. Mater., 2014, 26, 3526-3531.

35 B. S. Ghanem, R. Swaidan, E. Litwiller and I. Pinnau, Adv. Mater., 2014, 26, 3688-3692.

36 R. Swaidan, B. Ghanem, E. Litwiller and I. Pinnau, J. Membr. Sci., 2015, 475, 571-581. 
37 Q. Xie, J. Xu, L. Feng, L. Jiang, W. Tang, X. Luo and C. C. Han, Adv. Mater., 2004, 16, 302-305.

38 Q. Wang, Z. Cui, Y. Xiao and Q. Chen, Appl. Surf. Sci., 2007, 253, 9054-9060.

39 G.-Y. Li and Z.-G. Wang, Macromolecules, 2013, 46, 30583066.

40 C.-J. Shen, Y. Bao and Z.-G. Wang, Chem. Commun., 2013, 49, 3321-3323.

41 G.-Y. Li, B. Zhang, J. Yan and Z.-G. Wang, J. Mater. Chem. A, 2016, 4, 11453-11461.
42 S. A. Sydlik, Z. Chen and T. M. Swager, Macromolecules, 2011, 44, 976-980.

43 W. Ma, Q. Zhang, S. K. Samal, F. Wang, B. Gao, H. Pan, H. Xu, J. Yao, X. Zhan, S. C. De Smedt and C. Huang, RSC Adv., 2016, 6, 41861-41870.

44 Q. Zhang, S. Li, W. Li and S. Zhang, Polymer, 2007, 48, 62466253.

45 A. K. Gautam, C. Lai, H. Fong and T. J. Menkhaus, J. Membr. Sci., 2014, 466, 142-150.

46 B. Chakrabarty, A. Ghoshal and M. Purkait, J. Membr. Sci., 2008, 325, 427-437. 